\title{
Intravenous minocycline in multidrug-resistant infections: a profile of its use in the USA with a focus on Acinetobacter infections
}

\author{
Katherine A. Lyseng-Williamson ${ }^{1} \cdot$ Sarah L. Greig ${ }^{1} \cdot$ Lesley J. Scott $^{1}$
}

Published online: 7 November 2017

(C) Springer International Publishing AG, part of Springer Nature 2017

\begin{abstract}
Intravenous (IV) minocycline $\left(\right.$ Minocin $^{\circledR}$ ) is approved in the USA for use in patients with infections due to susceptible strains of many bacteria (e.g. Gram-positive and Gram-negative pathogens, including infections due to Acinetobacter spp.). Minocycline shows antibacterial activity against $A$. baumannii clinical isolates worldwide, and exhibits synergistic bactericidal activity against multidrug-resistant (MDR) and extensively drug-resistant (XDR) A. baumannii isolates when combined with other antibacterial agents. In retrospective studies, IV minocycline provided high rates of clinical success or improvement, and was generally well tolerated among patients with MDR or carbapenem-resistant $A$. baumannii infections.
\end{abstract}

\section{Adis evaluation of IV minocycline in susceptible infections}

Provides high rates of clinical success or improvement in patients with MDR or carbapenem-resistant Acinetobacter baumannii infections

Demonstrates antibacterial activity against $A$. baumannii clinical isolates (including MDR and XDR strains)

Exhibits synergistic bactericidal activity against $A$. baumannii isolates when combined with other antibacterials

Generally well tolerated

When used in combination with colistin, reduces the risk of colistinassociated nephrotoxicity relative to colistin alone

$I V$ intravenous, $M D R$ multidrug resistant, $X D R$ extensively drugresistant

Katherine A. Lyseng-Williamson

dtp@adis.com

1 Springer, Private Bag 65901, Mairangi Bay, 0754 Auckland, New Zealand

\section{What is the rationale for re-introducing the IV formulation of minocycline?}

Minocycline $\left(\operatorname{Minocin}^{\circledR}\right)$ is a second-generation tetracycline that has been available since the 1960s [1, 2]. An older intravenous (IV) formulation of minocycline was voluntarily withdrawn from the US market in 2005 due to declining use [2], and was reintroduced in 2009 to address the increase in MDR infections due to susceptible strains of Gram-positive and -negative pathogens, including infections due to Acinetobacter spp. [2, 3].

A new IV formulation of minocycline was approved by the US FDA in 2015 [4]. Trends in Acinetobacter baumannii resistance among clinical isolates from US patients with respiratory and blood stream infections have shown that, after the withdrawal of IV minocycline, the rate of minocycline resistance decreased from 56.5\% (2003-2005) to $30.5 \%$ (2009-2012), while resistance to other antibacterial agents (such as carbapenems and colistin) increased more than twofold [5]. Among A. baumannii isolates collected globally from integumentary sources in 2010-2014, the rate of minocycline resistance $(6.6 \%)$ was lower than that of any other antibacterial agent tested (30.9-50.3\%), and showed no significant change over the course of this time period [6]. These findings suggest a potential role for IV minocycline in treating patients with infections caused by A. baumannii and other susceptible Gram-positive and -negative bacteria.

\section{Why is it important to find antibacterials to treat A. baumannii?}

Acinetobacter spp. have recently emerged as a major cause of morbidity and mortality due to healthcare-associated infections, and are often multidrug-resistant (MDR) 
$[2,7,8]$. In recent years, the rates of $A$. baumannii resistance to almost all antibacterials (except minocycline) have risen in the USA [5]. Antibacterial therapy options are, therefore, becoming increasingly limited, particularly among patients with MDR or carbapenem-resistant $A$. baumannii infections.

Acinetobacter spp. are typically associated with respiratory and blood stream infections among critically ill patients, and MDR Acinetobacter spp. are considered a serious antibacterial resistance threat by the US Centers for Disease Control and Prevention [9]. A. baumannii is the most clinically relevant species within the Acinetobacter complex, although Acinetobacter nosocomialis and Acinetobacter pitti have also been associated with hospitalacquired infections [7]. Among A. baumannii clinical isolates collected from US patients in 2011-2014, the overall rate of MDR was $54.8 \%$, and varied by state (e.g. $5 \%$ in Oregon vs $88.1 \%$ in Puerto Rico) [10]. As treatment options for MDR A. baumannii become increasingly limited, particularly due to the lack of new antibacterial agents, the use of older drugs to treat this pathogen has been investigated $[1,2]$.

\section{For whom is IV minocycline indicated?}

IV minocycline is indicated in the treatment of infections caused by isolates of designated bacteria (e.g. Gram-positive and -negative pathogens, including Acinetobacter spp., as well as many other bacteria) when bacteriologic testing indicates appropriate susceptibility to the drug [4]. Table 1 provides a summary of the prescribing information of IV minocycline in the USA. Consult the US prescribing information [4] for further details on the designated bacteria for which IV minocycline is indicated.

\section{How should the susceptibility to minocycline be tested?}

Consistent with principles of good antimicrobial stewardship, minocycline should only be used to treat or prevent infections that are proven or strongly suspected to be caused by susceptible pathogens [4]. If available, in vitro culture and susceptibility information should be considered when selecting and modifying antibacterial therapy. If culture and susceptibility information is not available, local epidemiology and susceptibility patterns should be considered when determining the empiric selection of antibacterials [4].

According to current Clinical Laboratory Standards Institute (CLSI) and FDA breakpoints, minimum inhibitory concentrations (MICs) of $\leq 4 \mu \mathrm{g} / \mathrm{mL}$ indicate $A$. baumannii isolates are susceptible to minocycline, MICs of $8 \mu \mathrm{g} / \mathrm{mL}$ indicate A. baumannii isolates have intermediate susceptibility to minocycline, and MICs $\geq 16 \mu \mathrm{g} / \mathrm{mL}$ indicate $A$. baumannii isolates are resistant to minocycline [4].

In the international surveillance SENTRY study [11], the susceptibility to minocycline was higher than that to doxycycline and tetracycline (Table 2). This indicates that a surrogate class representative (i.e. tetracycline) should not be used to test for minocycline in vitro susceptibility. Testing should be performed directly using CLSI methods or validated commercial antimicrobial susceptibility test systems [11], such as broth or agar dilution or disk diffusion techniques. The accuracy of five standard minocycline susceptibility methods was compared using 107 carbapenem-resistant A. baumannii isolates [12]. All testing methods were associated with low rates of major susceptibility errors $(0.9 \%$ of isolates for all methods) and very major errors $(0-5.6 \%)$ for minocycline. However, rates of minor errors were high (14.0-37.4\%), usually due to overcalling strains that were susceptible to minocycline by reference testing methods as having intermediate susceptibility or resistance to minocycline using the other methods [12]. The highest major and minor error rates were both shown using the Etest with Mueller-Hinton agar (MHA) method, and the lowest major and minor error rates were both shown using the disk diffusion with MHA method [12].

The US prescribing information provides detailed information on the methods, interpretive criteria, and acceptable quality control ranges for susceptibility testing [4].

\section{What is the pharmacokinetic profile of minocycline?}

In healthy volunteers receiving a single IV dose of minocycline $200 \mathrm{mg}$, mean serum concentrations of minocycline were 4.18 and $1.38 \mu \mathrm{g} / \mathrm{mL}$ at the end of infusion and after $12 \mathrm{~h}$, respectively [4]. Following 3 days' administration of IV minocycline $100 \mathrm{mg}$ every $12 \mathrm{~h}$ or $200 \mathrm{mg}$ once daily, minocycline trough plasma concentrations were $1.4-1.8$ and $\approx 1 \mu \mathrm{g} / \mathrm{mL}$, respectively [4]. Due to its enhanced lipophilicity, the tissue penetration of minocycline is greater than that of tetracycline and doxycycline, with tissue:serum concentration ratios of $>1.0$ in the lung, liver, gallbladder and bile fluids, prostate, and other genitourinary organs [3].

Minocycline has at least six metabolites (some of which are active), is eliminated predominantly via the liver and hepatobiliary circulation, with $\approx 5-12 \%$ of a dose recovered in the urine and $20-35 \%$ in the feces, and a serum elimination half-life of $15-23 \mathrm{~h}$ following IV administration $[1,3,4]$. 
Table 1 Prescribing summary of intravenous minocycline (Minocin ${ }^{\circledR}$ ) in the treatment or prevention of infections that are proven or strongly suspected to be caused by susceptible bacteria in the USA [4]

\section{How is IV minocycline available, and how should it be reconstituted and stored?}

Availability

Storage before reconstitution/dilution

Reconstitution and dilution

Storage after dilution in IV bag

Single-use vials containing $100 \mathrm{mg}$ of sterile lyophilized minocycline powder Controlled room temperature $\left(20-25^{\circ} \mathrm{C} ; 68-77^{\circ} \mathrm{F}\right)$

Immediately further dilute in $100-1000 \mathrm{~mL}$ with sodium chloride, dextrose, or dextrose + sodium chloride injection USP, or in 250-1000 mL lactated Ringer's injection USP (do not dilute with calcium-containing solutions, as a precipitate may form)

Reconstitute with $5 \mathrm{~mL}$ sterile water for injection USP

Room temperature for up to $4 \mathrm{~h}$ or refrigerated $\left(2-8^{\circ} \mathrm{C} ; 36-46^{\circ} \mathrm{F}\right)$ for up to $24 \mathrm{~h}$

What is the administration regimen of IV minocycline?

Usual adult dose

Usual pediatric dose (children aged $>8$ years)

In whom is the use of IV minocycline contraindicated?

Patients who have shown hypersensitivity to any of the tetracyclines or to any of the components of the formulation

How should IV minocycline be used in special populations?

Patients with impaired renal function (exposure to tetracyclines may $\uparrow$ )

Patients with impaired hepatic function or taking other hepatotoxic drugs

Women who are, or become, pregnant during treatment

Women who are breastfeeding

Children aged $<8$ years
Initial dose of $200 \mathrm{mg}$, then $100 \mathrm{mg}$ administered over $60 \mathrm{~min}$ every $12 \mathrm{~h}$ and should not exceed $400 \mathrm{mg}$ in $24 \mathrm{~h}$ (e.g. initial doses of $200 \mathrm{mg}$, then 100 or $200 \mathrm{mg}$ every $12 \mathrm{~h}$ have been used)

Initial dose of $4 \mathrm{mg} / \mathrm{kg}$, then $2 \mathrm{mg} / \mathrm{kg}$ administered over $60 \mathrm{~min}$ every $12 \mathrm{~h}$, not to exceed the usual adult dose

What other special warnings and precautions pertain to the use of IV minocycline and other tetracyclines?

Permanent discoloration of teeth

Skeletal development (all tetracyclines form a stable calcium complex in any bone-forming tissue)

DRESS and hypersensitivity syndromes

Photosensitivity (exaggerated sunburn)

CNS-related adverse effects (e.g. light-headedness, dizziness, and vertigo)

\section{Clostridium difficile-associated diarrhea}

IH ( $\uparrow$ risk in women of childbearing age who are overweight or with IH history)
Azotemia, hyperphosphatemia, acidosis, and possible liver toxicity may occur Monitor levels of creatinine and BUN

Total daily dosage should not exceed $200 \mathrm{mg}$ in $24 \mathrm{~h}$

Use with caution (hepatotoxicity has been reported with minocycline)

Advise of the risk of fetal harm; tetracyclines cross the placenta and are found in fetal tissues, and can have toxic effects on the developing fetus (based on animal studies)

Discontinue breastfeeding or minocycline based on the importance of the drug to the woman

Use is not recommended unless the expected benefits outweigh the risks

Do not use during tooth development periods (i.e. last half of pregnancy and from infancy up to 8 years of age) unless other drugs are not likely to be effective or are contraindicated

$\downarrow$ In fibula growth rate seen in premature infants receiving oral tetracycline $25 \mathrm{mg} / \mathrm{kg}$ every $6 \mathrm{~h}$; reaction was reversible on drug discontinuation

Retardation of skeletal development may occur if tetracyclines are taken during early pregnancy (based on animal studies)

Discontinue use immediately if syndrome is recognized (may be fatal)

Has been reported in some individuals taking tetracyclines, including minocycline

Generally transient; usually rapidly disappear after discontinuation

If adverse events occur, caution patients about driving vehicles or using hazardous machinery

Consider as cause of diarrhea in all cases that occur after antibacterial use

If visual disturbance occurs, evaluate promptly (risk of permanent vision loss)

Monitor patients until they are stable (may take weeks after drug discontinuation)

What potential clinically relevant interactions may occur between IV minocycline and other drugs used in the hospital setting?

Anticoagulant therapy

Some patients may require a $\downarrow$ in anticoagulant dosage (tetracycline class can $\downarrow$ prothrombin activity)

Methoxyflurane

Concurrent use may result in fatal renal toxicity 
What are special warnings and precautions pertain to the inclusion of magnesium in the IV minocycline formulation?

Heart block or myocardial damage

Closely monitor patients with these cardiac conditions

Impaired renal function

Monitor levels of magnesium (excreted by the kidney) in patients with impaired function

Potentially serious drug interactions

May occur with CNS depressants, neuromuscular blocking agents and cardiac glycosides

DRESS drug rash with eosinophilia and systemic symptoms, $I H$ intracranial hypertension, $I V$ intravenous, $\uparrow$ increase(s/d); $\downarrow$ decrease(s/d)

Plasma concentrations of minocycline increased in a dose proportional manner as the minocycline dose increased (6-96 mg/kg/day) in an in vivo study in rabbits, with results that can be bridged to the human dose [13]. Increases in exposure of tissues to minocycline were also dose proportional. The results further suggested that minocycline could be active against susceptible target organisms in plasma, tissues, and other bodily fluids, as the antibacterial was highly distributed throughout the body [13]. In body tissues, concentrations of minocycline were highest in the liver, followed by the lungs, heart, spleen, kidney, brain and adipose tissue; in bodily fluids, concentrations were highest in the choroid, then epithelial lining fluid, pulmonary alveolar macrophages, vitreous humor, aqueous humor and cerebrospinal fluid [13].

\section{Pharmacodynamic/pharmacokinetic considerations}

The efficacy of minocycline correlated with the area under the plasma concentration-time curve (AUC):MIC ratio in animal models of $A$. baumannii pneumonia [14, 15]. For example, the 24-h free AUC:MIC (fAUC:MIC) ratios for minocycline were $10.6-16.1$ and $13.1-24.2$ for bacteriostatic and bactericidal effects, respectively [14]. The exposure to minocycline was equivalent to dosages of $200-400 \mathrm{mg} /$ day in humans [14], thereby supporting the importance of ensuring that adequate dosages of IV minocycline are administered to maximize efficacy.

Single compartment dilutional pharmacokinetic models of A. baumannii infection have been used to determine the in vitro relationship between exposure to minocycline and antibacterial effect $[16,17]$. In one model, minocycline exhibited bactericidal activity (i.e. $-1 \log _{10}$ drop) against A. baumannii at fAUC:MIC ratios of 23.2 at $24 \mathrm{~h}$, and 30.4 at $48 \mathrm{~h} \mathrm{[16].} \mathrm{Based} \mathrm{on} \mathrm{this} \mathrm{model,} \mathrm{an} f$ AUC:MIC ratio target of 15-20 was considered reasonable for minocycline against $A$. baumannii [16]. In another model [17], fAUC:MIC ratios at $24 \mathrm{~h}$ were 16.4 and 23.3 for bacteriostatic and bactericidal effects against $A$. baumannii, respectively, suggesting a reasonable $f$ AUC:MIC ratio target of 20-25. As resistance emerged at $f$ AUC:MIC ratios of 5-15, suggesting that combination treatment with minocycline + another antibacterial, or an increase of minocycline dosage to $>400 \mathrm{mg} / \mathrm{day}$, should be considered when treating A. baumannii strains, in order to reduce the emergence of resistance [17].

\section{What is the pharmacodynamic profile of minocycline?}

Minocycline is a semisynthetic derivative of tetracycline that is thought to exert its primarily bacteriostatic effects through inhibition of protein synthesis [4]. Like other tetracyclines, minocycline reversibly binds to the bacterial ribosomal $30 \mathrm{~S}$ subunit, which results in conformation changes in the $16 \mathrm{~S}$ ribosomal RNA that inhibit the association of aminoacyl transfer RNA with the ribosome, and ultimately disrupt bacterial protein synthesis $[1,3]$. Of note, minocycline may also have immunomodulatory effects in the treatment of A. baumannii infection [18]. According to a recent in vitro study [18], minocycline reduced the production of inflammatory cytokines in macrophages, in addition to enhancing their antibacterial activity.

\section{Antibacterial activity in vitro}

The in vitro antibacterial activity of minocycline against clinical isolates of $A$. baumannii (including MDR A. baumannii isolates) based on the MIC required to inhibit growth in $90 \%$ of isolates $\left(\mathrm{MIC}_{90}\right)$ has been shown in ongoing global and US surveillance studies (Table 2) [11, 19-23].

In TEST (2004-2013) [19], minocycline MIC $_{90}$ values were 8 and $16 \mu \mathrm{g} / \mathrm{mL}$ against $A$. baumannii and MDR $A$. baumannii isolates, respectively. These values are consistent with those in other surveillance studies including:

- A regional update of TEST (2011-2014) [24] Minocycline $\mathrm{MIC}_{90}$ values against MDR A. baumannii were $8 \mu \mathrm{g} / \mathrm{mL}$ in Africa, Asia-Pacific, Latin America, and North America, and $16 \mu \mathrm{g} / \mathrm{mL}$ in Europe and the Middle East.

- Isolates collected from global integumentary sources (2010-2014) [6] Minocycline $\mathrm{MIC}_{90}$ value of $8 \mu \mathrm{g} / \mathrm{mL}$ against $A$. baumannii $(n=1235 ; 43.2 \%$ of which were MDR). 
Table 2 In vitro activity of minocycline against Acinetobacter baumannii isolates collected internationally in 2004-2013 (TEST [19, 20, 22, 23], SENTRY [11] and a global surveillance programme [21])

\begin{tabular}{|c|c|c|c|c|}
\hline \multirow[t]{2}{*}{ Isolate (no. of isolates) } & \multirow{2}{*}{$\begin{array}{l}\mathrm{MIC}_{90} \\
(\mu \mathrm{g} / \mathrm{mL})\end{array}$} & \multicolumn{2}{|l|}{ Isolates $(\%)^{\mathrm{a}}$} & \multirow{2}{*}{$\begin{array}{l}\text { Other results/comments regarding the susceptibility of } \\
\text { A. baumannii isolates }\end{array}$} \\
\hline & & Susceptible & Resistant & \\
\hline \multicolumn{5}{|c|}{ Global (2004-2013) [11, 19-21] } \\
\hline \multirow[t]{3}{*}{$\begin{array}{l}\text { A. baumannii } \\
(1312-16,778) \\
{[11,19-21]}\end{array}$} & \multirow[t]{3}{*}{$\geq 8[11,19-21]$} & \multirow[t]{3}{*}{$\begin{array}{l}72.3-84.5 \\
\quad[11,19-21]\end{array}$} & \multirow[t]{3}{*}{$\begin{array}{l}5.1-12.6 \\
\quad[19-21]\end{array}$} & $\begin{array}{l}\text { Susceptibility to minocycline was higher than that of } \\
\text { doxycycline and tetracycline ( } 79.1 \text { vs } 59.6 \text { and } 30.2 \% \\
\text { of isolates); } 98.8 \% \text { of isolates were susceptible to } \\
\text { colistin (MIC } 901 \mu \mathrm{g} / \mathrm{mL} \text { ) and } 80.7 \% \text { of isolates were } \\
\text { inhibited by tigecycline } \leq 1 \mu \mathrm{g} / \mathrm{mL} \text { [11] }\end{array}$ \\
\hline & & & & MIC range: $\leq 0.5$ to $\geq 32 \mu \mathrm{g} / \mathrm{mL}[19]$ \\
\hline & & & & $\mathrm{MIC}_{50}: 2 \mu \mathrm{g} / \mathrm{mL}[21]$ \\
\hline \multirow[t]{3}{*}{$\begin{array}{l}\text { MDR A. baumannii } \\
(1070-6743) \\
{[19-21]}\end{array}$} & \multirow[t]{3}{*}{$>8-16$ [19-21] } & \multirow[t]{3}{*}{$66.2-75.4[19-21]$} & \multirow[t]{3}{*}{$\begin{array}{l}8.3-15.4 \\
\quad[19-21]\end{array}$} & $\begin{array}{l}\text { Susceptibility rates with other antibacterials were } \\
\text { generally more than twofold lower in MDR isolates } \\
\text { than in non-MDR isolates [19] }\end{array}$ \\
\hline & & & & MIC range: $\leq 0.5$ to $\geq 32 \mu \mathrm{g} / \mathrm{mL}[19]$ \\
\hline & & & & $\mathrm{MIC}_{50}: 4 \mu \mathrm{g} / \mathrm{mL}[21]$ \\
\hline $\begin{array}{l}\text { XDR A. baumannii } \\
\text { (943) [21] }\end{array}$ & $>8[21]$ & $62.9[21]$ & $16.9[21]$ & $\mathrm{MIC}_{50}: 4 \mu \mathrm{g} / \mathrm{mL}[21]$ \\
\hline
\end{tabular}

USA (2005-2011) [Pacific, Mountain, West North Central, East North Central, Middle Atlantic, New England, South Atlantic, East South Central, and West South Central regions] [22]

\begin{tabular}{|c|c|c|c|c|}
\hline \multirow{2}{*}{$\begin{array}{l}\text { A. baumannii }(2900 \\
\text { across USA; } 72-721 \\
\text { in each region) }\end{array}$} & Across USA:8 & Across USA: 84.1 & \multirow[t]{2}{*}{ NR } & $\mathrm{MIC}_{50}$ across USA: $\leq 0.5 \mu \mathrm{g} / \mathrm{mL}$ \\
\hline & By region: $4-8$ & By region: $68.5-97.4$ & & $\mathrm{MIC}_{50}$ by region: $\leq 0.5-2 \mu \mathrm{g} / \mathrm{mL}$ \\
\hline \multirow{2}{*}{$\begin{array}{l}\text { MDR A. baumannii } \\
\text { (883) }\end{array}$} & Across USA: 8 & Across USA: 72.1 & \multirow[t]{2}{*}{ NR } & $\mathrm{MIC}_{50}$ across USA: $2 \mu \mathrm{g} / \mathrm{mL}$ \\
\hline & By region: $4-16$ & By region: $54.0-92.3$ & & $\mathrm{MIC}_{50}$ by region: $1-4 \mu \mathrm{g} / \mathrm{mL}$ \\
\hline
\end{tabular}

Pediatric patients (2004-2012) [23]

A. baumannii (1302) $4 \quad 90.8 \quad$ NR
Isolates from patients aged 1-5 years had significantly higher minocycline susceptibility rates than those from patients aged $\geq 18$ years $(92.0$ vs $84.6 \%$; $p=0.0001)$
Minocycline $\mathrm{MIC}_{90}$ values were lower in pediatric isolates than in the overall population (4 vs $\geq 8$ )
Global rate of MDR A. baumannii isolates: $19.5 \%$ (5.6, 51.1 and $52.5 \%$ in North America, Latin America and the Middle East, respectively)

$C L S I$ Clinical and Laboratory Standards Institute, $M D R$ multidrug-resistant (i.e. nonsusceptible to $\geq 1$ agent in $\geq 3$ antibacterial classes), $M I C$ minimum inhibitory concentration (determined using the CLSI broth microdilution method), $M I C_{50}$ MIC required to inhibit growth in $50 \%$ of isolates, $M I C_{90}$ MIC required to inhibit growth in $90 \%$ of isolates, $N R$ not reported, $X D R$ extensively drug-resistant (i.e. nonsusceptible to $\geq 1$ agent in all but $\leq 2$ antibacterial classes)

${ }^{a}$ Using CLSI breakpoints indicating susceptibility, intermediate susceptibility, and resistance to minocycline (i.e. $\leq 4,8$, and $\geq 16 \mu \mathrm{g} / \mathrm{mL}$, respectively)

- Isolates collected from patients in intensive care units (2004-2010) [20] Minocycline $\mathrm{MIC}_{90}$ value of $8 \mu \mathrm{g} /$ $\mathrm{mL}$ for both $A$. baumannii $(n=4241 ; 58.7 \%$ of which were MDR) and MDR A. baumannii isolates $(n=2491)$.

Susceptibility to minocycline remained consistent among A. baumannii clinical isolates collected in 2004-2013 in the global and US surveillance studies (Table 2) [11, 19-23].

\section{Bactericidal activity in vitro and in vivo}

Minocycline + other antibacterials demonstrated synergistic bactericidal activity against clinical A. baumannii [25], MDR A. baumannii [26, 27], and extensively drug-resistant (XDR) A. baumannii [28] isolates in vitro. When used alone, minocycline was bacteriostatic against MDR A. baumannii isolates at concentrations achieved with therapeutic dosages, and bactericidal when combined with colistin [27], rifampicin [26, 27], imipenem [27], erythromycin [26], amikacin 
[26], or polymyxin B [26]. In isolates not harbouring the tet $B$ gene, bactericidal effects were observed with minocycline $2-8 \mu \mathrm{g} / \mathrm{mL}+$ colistin, and minocycline $0.5-8 \mu \mathrm{g} /$ $\mathrm{mL}+$ rifampicin or imipenem [27]. Minocycline also inhibited the growth of XDR A. baumannii isolates when used alone at a concentration $4 \mu \mathrm{g} / \mathrm{mL}$, with synergistic bactericidal activity being shown with minocycline $4 \mu \mathrm{g} /$ $\mathrm{mL}+$ meropenem at $12 \mathrm{~h}$, and minocycline $2 \mu \mathrm{g} /$ $\mathrm{mL}+$ colistin within 2-6 h [28].

Against minocycline-resistant isolates of A. bauman$n i i$, minocycline + colistin also displayed synergistic activity against all isolates of minocycline-resistant $A$. baumannii, with the combination being more effective than other minocycline-based combinations, and as effective as meropenem + colistin [29]. The combination of polymyxin $\mathrm{B}$ and minocycline also shown bactericidal efficacy against Klebsiella pneumoniae carbapenemase-producing $K$. pneumonia in a preliminary in vitro study [30].

Consistent with the vitro activity of minocycline, it displayed bactericidal activity in vivo [14, 15, 25, 26, 31]. In neutropenic animal models of $A$. baumannii pneumonia, minocycline displayed bactericidal activity $[14,31]$, and reduced 24-h bacterial tissue burden when used as monotherapy [15, 25, 31]. Reductions in 24-h bacterial tissue burden were greater when minocycline was used in combination with polymyxin B [25], and lung tissue inflammatory infiltration reduced to a greater extent with minocycline + rifampicin or amikacin than with polymyxin B alone [26].

\section{Resistance issues}

Resistance to tetracycline is commonly due to the acquisition of genes that encode efflux pumps [11]. Of the six major facilitator superfamily efflux pumps detected in Acinetobacter spp., TetB is the only one capable of transporting minocycline from bacteria [11]. The tetB gene was detected in all XDR A. baumannii isolates with minocycline resistance collected in Argentina (1983-2011); all tet $B$-positive isolates had a plasmid-borne ISCR 2 element, which may help explain the spread of minocycline resistance in Acinetobacter spp. [32]. The widespread prevalence of the tetB::ISCR2 resistance element in XDR $A$. baumannii isolates was confirmed in isolates collected in Argentina in 2009-2013 [33].

Most tetB-negative $A$. baumannii isolates are susceptible to minocycline, whereas tetB-positive isolates have lower susceptibility rates $[34,35]$. In a study using 258 clinical isolates of $A$. baumannii collected world-wide in 1998-2015, resistance to minocycline, doxycycline, levofloxacin, and meropenem were shown in 44.6, 68.8, 93.0, and $80.6 \%$ of isolates, respectively [34]. MIC values for minocycline had a high degree of separation between the 93 tet $B$-negative isolates and the 165 tet $B$-positive isolates $(\leq 0.0625-4$ vs $4-32 \mu \mathrm{g} / \mathrm{mL}$ ); the MIC of almost all $(93 \%)$ tetB-positive isolates were $>4 \mu \mathrm{g} / \mathrm{mL}$ (i.e. higher than the susceptibility breakpoint for minocycline), whereas all tetB-negative isolates had MIC values lower than this breakpoint [34]. In another study using 107 carbapenem-resistant $A$. baumannii isolates, all tet $B$-negative and $71.1 \%$ of $t e t B$-positive isolates were susceptible to minocycline, with median minocycline MICs of 4 (range $0.125-16) \mu \mathrm{g} / \mathrm{mL}$ and 1 (range $\leq 0.06-2$ ) $\mu \mathrm{g} / \mathrm{mL}$, respectively [35]. These findings suggest that testing for the presence of tet $B$ may be a rapid surrogate method for determining susceptibility to minocycline (i.e. the absence of $t e t B$ predicts susceptibility to minocycline), with further studies being warranted [34].

The addition of polymyxin B 0.25 or $0.5 \mu \mathrm{g} / \mathrm{mL}$ enhances the activity of minocycline against tetB-positive $A$. baumannii isolates, including those resistant to polymyxin B, by a factor of 2-154 [36]. In a study using 167 clinical isolates of tetB-positive A. baumannii isolates, including 4 resistant to polymyxin $\mathrm{B}$, only $12.0 \%$ of isolates were susceptible (i.e. MIC $\leq 4 \mu \mathrm{g} / \mathrm{mL}$ ) to minocycline alone (MIC $0.5-32 \mu \mathrm{g} / \mathrm{mL}$ ) [36]. The addition of polymyxin B $0.125,0.25$, or $0.5 \mu \mathrm{g} / \mathrm{mL}$ increased susceptibility rates to $18.0,58.1$, and $100 \%$, respectively, with corresponding MIC values of $0.5-16,0.5-16$, and $\leq 0.06-4 \mu \mathrm{g} / \mathrm{mL}$. In the isolates resistant to polymyxin $\mathrm{B}$, MIC values were $8-32 \mu \mathrm{g} / \mathrm{mL}$ with minocycline alone and $2-4 \mu \mathrm{g} / \mathrm{mL}$ with minocycline + polymyxin B $0.5 \mu \mathrm{g} /$ $\mathrm{mL}$ [36]. Clinical studies of the use of minocycline + polymyxin $\mathrm{B}$ in the treatment of A. baumannii infections, especially those resistant to one or both of these antibacterials, would be of interest.

Several efflux pump systems from the resistance-nodulation-cell division family are also associated with MDR $A$. baumannii [11, 37]. In clinical isolates of MDR A. baumannii collected in Spain (2010), overexpression of the AdeABC efflux pump system was associated with increased MIC values for minocycline $(>2 \mu \mathrm{g} / \mathrm{mL})$, tigecycline $(>0.5 \mu \mathrm{g} / \mathrm{mL})$, and gentamycin $(>8 \mu \mathrm{g} / \mathrm{mL})$, while overexpression of the AdeIJK efflux pump (alone or together with TetB) was associated with increased minocycline MIC values $(\geq 2 \mu \mathrm{g} / \mathrm{mL})$ [37].

In vitro, a few intrinsic mechanisms may modestly elevate minocycline MIC values, with these values remaining within the $A$. baumannii susceptible range $[38,39]$. Mutations in minocycline-resistant strains have been located in adeN (a negative regulator of the AdeIJK 
efflux pump), trm ( $S$-adenosyl methionine-dependent methyltransferase), and/or the $\approx 720 \mathrm{bp}$ region between the $r l u C$ and rne genes [38]. The maximum minocycline MIC ( $4 \mu \mathrm{g} / \mathrm{mL})$ in these mutants was lower or at the current CLSI susceptibility breakpoint [38]. The minocycline mutant prevention concentration $(1 \mu \mathrm{g} / \mathrm{mL})$ was below the minocycline peak and trough plasma concentrations obtained with the usual dosage [39]. Of note, unlike tigecycline, minocycline is not a substrate for the adeABC efflux system [39].

\section{What is the effectiveness of IV minocycline in Acinetobacter infections?}

Given the difficulty of performing large prospective trials in less common pathogens such as Acinetobacter spp., prospective randomized clinical trials of IV minocycline have not been conducted. Moreover, such trials were not required by the FDA when IV minocycline was first approved several decades ago. Nevertheless, several retrospective single-centre studies have documented the effectiveness of IV minocycline in the treatment of infections caused by $A$. baumannii and other pathogens, MDR A. baumannii [40-42], and carbapenem-resistant A. baumannii $[43,44]$ in the clinical practice setting in the USA.

Treatment with IV minocycline was associated with clinical improvement or treatment success in small studies in hospitalized patients with MDR [40-42] or carbapenemresistant A. baumannii [43, 44] (Table 3). In the largest of these studies, treatment with IV minocycline achieved clinical success in $73 \%$ of patients with MDR A. baumannii infections (Table 3) [40], most commonly respiratory, blood, and respiratory + blood infections $(58,18$, and $7 \%$ of patients, respectively). [40]. During the 30-day followup period, one patient (who had achieved presumed microbiologic eradication with minocycline + colistin for 14 days) was readmitted at 16 days post-discharge with worsening respiratory status due to an MDR A. baumannii infection. Upon readmission, the minocycline MIC had increased from 4 to $6 \mathrm{mg} / \mathrm{L}$ (i.e. intermediate susceptibility) on MDR A. baumannii respiratory culture. The patient achieved clinical success following treatment with colistin + doripenem for 14 days [40].

Limited data suggest that minocycline is also an effective option for treating carbapenem-resistant $K$. pneumonia infection [45]. In a case series of four patients with carbapenem-resistant $K$. pneumonia infection in one US hospital, all patients achieved a good clinical response with twice-daily oral minocycline $200 \mathrm{mg}$, with one patient developing further carbapenem-resistant bacteremia 18 days after completion of the initially successful therapy [45].
What is the tolerability profile of IV minocycline?

IV minocycline is generally well tolerated when used to treat infections. No adverse events were considered to be related to treatment with IV minocycline in retrospective studies in patients with MDR or carbapenem-resistant $A$. baumannii infections [40, 41, 43]. In one of these studies, a patient with carbapenem-resistant A. baumannii pneumonia developed acute kidney injury, which was presumed to be related to the use of concomitant colistin [43]. According to a review of adverse event data in 84 patients with MDR A. baumannii, neutropenia + eosinophilia was reported in one minocycline recipient [46].

The tetracycline class of antibacterials are commonly associated with gastrointestinal effects (e.g. nausea, anorexia, and diarrhea), CNS effects (e.g. dizziness, lightheadedness, and vertigo), fever, permanent tooth discolouration, photosensitivity and other dermatologic conditions, liver and renal toxicity, hypersensitivity, and respiratory, genitourinary, musculoskeletal and blood disorders [4]. When administered via IV, local reactions (e.g. injection-site erythema or pain) may occur; tinnitus and decreased hearing have also been reported in recipients of IV minocycline [4]. Appropriate precautions should be following to minimize the risk and severity of adverse events (Table 1) [4].

\section{How is IV minocycline being used in the hospital setting?}

Based on data extracted from the Premier Research database (a large database of $>500$ US hospitals), a retrospective study analysed the patterns of use of IV minocycline in the hospital setting [47]. The study included 521 inpatients in 44 US hospitals who received at least one dose of IV minocycline during an 18-month study period (1 Jan 2014 to 30 Jun 2015). Patients had a mean age of 61.8 years, and frequently had comorbid conditions (29.2-36.8\% of patients had chronic pulmonary disease, renal disease, congestive heart failure, and/or diabetes). The mean Charlson Comorbidity Index score of minocycline recipients was 3.17 [47], which is considered relatively high (a score of $\geq 5$ essentially indicates a $100 \%$ risk of dying at 1 year) [48].

The most common primary International Classification of Diseases, 9th edition (ICD-9) diagnoses for which patients received IV minocycline were 'infectious and parasitic diseases' and 'respiratory system diseases' (28.1 and $19.4 \%$ of patients, respectively) [47]. The primary infections most frequently treated with IV minocycline were septicemia (26.7\% of patients), complications of 
Table 3 Efficacy of intravenous minocycline in adult patients with Acinetobacter baumannii infections in retrospective single-center studies
Type of infection (study
Relevant patient population and treatment regimens
Main clinical outcomes

dates)

\section{MDR A. baumannii}

MDR A. baumannii [40]

(Sep 2010-Mar 2013)
All 55 pts had MDR A. baumannii infections (nonsusceptible to $\geq 1$ agent from $\geq 3$ antibacterial classes) with cultures susceptible to minocycline (i.e. MIC $\leq 4 \mu \mathrm{g} / \mathrm{mL}$ )

Pts received IV minocycline $100 \mathrm{mg}$ twice daily within $72 \mathrm{~h}$ of the onset of infection for $\geq 48 \mathrm{~h}$ (median treatment duration 9 days); most (76\%) pts also received a $200 \mathrm{mg}$ loading dose

All but 3 pts received $\geq 1$ other concomitant antibacterial agent, most commonly colistin $(n=45)$, doripenem $(n=20)$, and ampicillin/sulbactam $(n=17)$

MRSA or MDR Gramnegative bacteria [41] (Nov 2009-Apr 2012)

MDR A. baumannii VAP [42] (Jan-Dec 1998)
5/21 pts had MDR A. baumannii infections and received minocycline $100 \mathrm{mg}$ every $12 \mathrm{~h}$

\section{$4 / 7$ pts received minocycline $100 \mathrm{mg}$ every $12 \mathrm{~h}$} $(+$ imipenem/cilastatin in $1 \mathrm{pt} ;+$ trovafloxacin + trimethoprim/sulfamethoxazole in $1 \mathrm{pt}$ ); $3 / 7$ received doxycline $100 \mathrm{mg}$ every $12 \mathrm{~h}$

\section{Carbapenem-resistant $\boldsymbol{A}$. baumannii}

Carbapenem-resistant

Gram-negative bacteria [43] (not reported)
7/9 pts had carbapenem-resistant A. baumannii infections and received twice-daily IV minocycline 100 or $200 \mathrm{mg}(n=2$ and 5$) \times$ 4-13 days; 6 pts also received colistin, meropenen, and/or ampicillin/sulbactam $(n=5,1$, and 1$)$

$19 / 55$ pts received IV minocycline (200 mg loading dose, then $100 \mathrm{mg}$ twice daily) or IV doxycycline
$73 \%$ of patients achieved clinical success (defined as complete or partial resolution of MDR $A$.

baumannii infection signs and symptoms without the need for escalated antibacterial treatment; primary outcome) with minocycline

$27 \%$ pts had clinical failure (i.e. persistent signs and symptoms of infection with the need for additional antibacterial agents); a total of $78 \%$ of pts achieved documented or presumed microbiologic eradication with minocycline

Overall rate of $A$. baumannii infection-related mortality was $25 \%$ (14 deaths; 12 from pneumonia and 2 from pneumonia + bacteremia); median hospital length of stay was 31 days; median infection-related hospital length of stay was 16 days

All pts with MDR A. baumannii infections showed clinical improvement with minocycline

All (4/4) minocycline and 2/3 doxycycline recipients achieved treatment success (defined as the absence of A. baumannii from follow-up bronchoalveolar lavage culture and/or improvement in clinical symptoms)

$5(71 \%)$ patients with carbapenem-resistant $A$. baumannii infections achieved clinical cure (defined as resolution of signs and symptoms of infection) with minocycline

$3 / 5$ pts in whom repeat cultures were reported achieved microbiologic cure

2 pts without clinical or microbiologic cure subsequently died

In the combined minocycline/doxycycline groups, $79 \%(15 / 19)$ achieved a clinical response (defined as improvement and resolution of VAP or microbiologic eradication of $A$. baumannii) baumannii VAP [44]

(Jul 2004 to Dec 2007)

IV intravenous, MDR multidrug-resistant, MRSA methicillin-resistant Staphylococcus aureus, pt(s) patient(s), VAP ventilator-associated pneumonia

device, implant, procedure, or medical care $(7.7 \%)$, skin and subcutaneous tissue infection (6.9\%), pneumonia $(6.3 \%)$, and respiratory failure (5.8\%) [47].

Overall, the patient population receiving IV minocycline was severely ill, with $65.5 \%$ of patients being treated in ICU, and $54.1 \%$ requiring the use of mechanical ventilation [47]. Almost all patients $(\approx 91 \%)$ required immediate admission to hospital, including $74.9 \%$ who required emergency admission and $15.4 \%$ who required urgent admission [47].

The use of IV minocycline in US hospitals appears to be increasing substantially over time [47]. In the 44 US hospitals included in the retrospective study of the
Premier Research database, $<50$ patients received IV minocycline in 2009 ; however, $\approx 400$ patients were projected to receive it in 2015 [47]. The mean overall length of IV minocycline treatment was 5.4 days, with most $(68.3 \%)$ patients receiving treatment for $\geq 3$ days. On the first day of administration, $21.3 \%$ of patients received $100 \mathrm{mg}$ of IV minocycline, $44.5 \%$ received $200 \mathrm{mg}, 25.1 \%$ received $300 \mathrm{mg}$, and $7.9 \%$ received $400 \mathrm{mg}$; a total of $69.6 \%$ received a first-day loading dose of $200 \mathrm{or} 300 \mathrm{mg}$. For the remainder of treatment, the majority of patients received $200 \mathrm{mg}$ of IV minocycline per day (e.g. 75.3 and $74.4 \%$ of patients on days 2 and 3 of treatment) [47]. 
In a breakdown of mean hospital costs per IV minocycline recipient in the survey, the cost of IV minocycline (\$US1108) accounted for $5.7 \%$ of the total pharmacy costs, which in turn accounted for $20 \%$ (\$US19,453) of the total per patient cost of \$US96,450 [47]. Room + board was the leading single cost component (\$US35,347; 37\% of the total costs); operating room costs (\$US15,258) accounted for $16 \%$ of total costs, with all other cost categories each accounting for $<1-7 \%$ of the total costs [47].

\section{Does adding minocycline reduce the risk of polymyxin-associated toxicity?}

Minocycline and polymyxins (e.g. colistin) display synergistic antibacterial activity [27, 28, 36], and may be used in combination to treat MDR A. baumannii infections. However, polymyxins are well known to have nephrotoxic and neurotoxic effects, even at the plasma concentrations needed for bactericidal activity [49, 50]. The addition of minocycline to polymyxin treatment may potentially ameliorate polymyxin-associated nephrotoxicity and neurotoxicity, in addition to its synergistic antibacterial activity. According to a recent systematic review of the potential mechanism of minocycline in kidney diseases [49], minocycline provides protection against the development and progression of kidney disease by suppressing apoptosis, scavenging free radicals, scavenging, preventing inflammation and mitochondrial dysfunction, and inhibiting matrix metalloproteinase. Moreover, in an in vitro study, minocycline provided protective effects against colistin-associated neurotoxicity by scavenging reactive oxygen species and suppressing apoptosis [50].

Two retrospective cohort studies (based on data extracted from the Premier Research database) analysed the occurrence of acute renal failure (ARF) in adult patients admitted to an ICU with an ICD-9 diagnosis of pneumonia or sepsis who had received IV colistin for $\geq 3$ days during the study period (1 Jan 2010 to 31 Dec 2015) [51, 52].

One analysis included a total of 4602 IV colistin recipients, with 3512 receiving colistin alone, 95 colistin + minocycline (including those who received both minocycline and tigecycline), and 995 colistin + tigecycline (an overlap period of $\geq 3$ days was required for combination therapy) [51]. The unadjusted rate of colistinassociated ARF when colistin was used alone (21.2\% of patients) significantly decreased when colistin minocycline was administered [11.6\%; $p=0.003$; OR 0.49 (95\% CI 0.26-0.92)], but significantly increased when colistin was used with tigecycline $[25.6 \% ; p=0.024$; OR $1.28(95 \%$ CI 1.09-1.51)]. In addition, the unadjusted mortality rate did not differ significantly between colistin alone and colistin + minocycline (27.0 vs $31.6 \%$ of patients), but was significantly higher with colistin + tigecycline $(35.4 \%)$ than that with colistin alone $[p<0.001$; OR 1.48 (95\% CI 1.28-1.72)] [51]. There were no significant between-group differences with regard to unadjusted rates of 30-day hospital readmission (26.6 vs 30.8 and $26.4 \%$ of colistin alone, colistin + minocycline, and colistin + tigecycline recipients, respectively) [51].

Similar results with colistin + minocycline versus colistin alone were shown in the other analysis, which included a total of 5120 patients with 5025 receiving colistin alone and 95 colistin + minocycline (median duration of overlap therapy 7.36 days). Colistin was initiated before, concomitantly, and after minocycline in 45.3, 35.8, and $18.9 \%$ of patients, respectively) [52]. The rate of colistinassociated ARF was significantly lower with colistin + minocycline than with colistin alone based on unadjusted data [11.6 vs 23.0\%; $p=0.009$; OR $0.48(95 \%$ CI $0.23-0.83$ )] and adjusted (propensity score matching) data $[11.6$ vs $24.7 \% ; p=0.007$; OR $0.40 \quad(95 \% \quad \mathrm{CI}$ $0.20-0.79)]$. There were no significant between-group differences with regard to either unadjusted or adjusted rates of in-hospital mortality or 30-day readmission. Based on conventional regression analysis, the development of ARF significantly $(p<0.001)$ increased per-patient hospital costs (additional \$US10,308) and hospital length of stay (additional 3.4 days) in this study [52].

\section{What conclusions can be made regarding the clinical use of IV minocycline?}

IV minocycline is an option in the treatment of patients with susceptible MDR A. baumannii infections, particularly when used with a second antibacterial agent, such as colistin or polymyxin B. It may also be used to treat other difficult-to-treat infections caused by susceptible bacteria. Retrospective studies in the clinical-practice setting have shown IV minocycline to be generally effective and well tolerated in patients with MDR [40-42] or carbapenemresistant A. baumannii [43, 44] infections. IV minocycline is also a re-emerging option in the treatment of susceptible carbapenem-resistant infections caused by the Enterobacteriaceae family of Gram-negative bacteria (e.g. Escherichia coli, K. pneumoniae, and Enterobacter spp.), which is a common and increasingly resistant source of infection [30, 53].

Analyses of data from the Premier Research database are helpful in showing how IV minocycline is being used as mono- and combination therapy in the clinical-practice setting in the USA [47, 51, 52]. However, as the data in these analyses were derived from administrative records, only very limited information regarding causative pathogens and treatment outcomes are available. Despite these 
limitations, the analyses have indicated that there is an increasing need for clinically effective antibacterials, such as minocycline, to treat MDR A. baumannii and other serious infections, and that the addition of IV minocycline, but not IV tigecycline, to colistin treatment may reduce the risk of colistin-associated acute renal failure.

The use of IV minocycline may also be cost effective in the treatment of MDR A. baumannii infections from a US hospital perspective, according to the results of a decisiontree model [54]. IV minocycline monotherapy was predicted to be cost saving relative to meropenem monotherapy for the treatment of MDR A. baumannii after a positive culture (incremental gain of 3.38 life-years (LYs), and a decrease in hospital-related costs of \$US2099 (2014 values)]. After the failure of carbapenem therapy, a switch to IV minocycline was estimated to be a cost saving relative to a switch to colistin (incremental gain of 3.12 LYs, and decrease in cost of \$US1599), and cost effective relative to tigecycline (incremental cost per LY gained of \$415) [54]. However, the results of this analysis are limited by the incorporation of assumed clinical outcomes, which was necessary due to the lack of robust comparative clinical data, and the simplistic model design, which considered only the use of antibacterial monotherapy instead of both mono- and combination therapy [54].

Head-to-head comparative clinical and pharmacoeconomic studies would help clarify the position of IV minocycline relative to other antibacterials in the treatment of susceptible MDR and XDR A. baumannii infections.

Acknowledgements The manuscript was updated from Drugs 2016;76(15):1467-76 [55], and was reviewed by: D.R. Abernethy, Office of Clinical Pharmacology, US Food and Drug Administration, Silver Spring, MD, USA; M. Morgado, CICS-UBI Health Sciences Research Centre, University of Beira Interior, Covilhã, Portugal. During the peer review process, the marketing authorization holder of IV minocycline was offered an opportunity to review this article. Changes resulting from comments received were made on the basis of scientific and editorial merit.

\section{Compliance with ethical standards}

Funding The preparation of this review was not supported by any external funding.

Conflict of interest K.A. Lyseng-Williamson, S.L. Greig SL, and L.J. Scott are employees of Adis/Springer, are responsible for the article content and declare no conflicts of interest.

Additional information about this Adis Drug Q\&A can be found here http://www.medengine.com/Redeem/F1CCF060601D5A39

\section{References}

1. Ritchie DJ, Garavaglia-Wilson A. A review of intravenous minocycline for treatment of multidrug-resistant Acinetobacter infections. Clin Infect Dis. 2014;59(Suppl 6):S374-80.
2. Bishburg E, Bishburg K. Minocycline: an old drug for a new century: emphasis on methicillin-resistant Staphylococcus aureus (MRSA) and Acinetobacter baumannii. Int J Antimicrob Agents. 2009;34(5):395-401.

3. Colton B, McConeghy KW, Schreckenberger PC, et al. I.V. minocycline revisited for infections caused by multidrug-resistant organisms. Am J Health Syst Pharm. 2016;73(5):279-85.

4. Minocin ${ }^{\circledR}$ (minocycline for injection) $100 \mathrm{mg} /$ vial: US prescribing information. Parsippany (NJ): The Medicines Company; 2015.

5. Zilberberg MD, Kollef MH, Shorr AF. Secular trends in Acinetobacter baumannii resistance in respiratory and blood stream specimens in the United States, 2003 to 2012: a survey study. J Hosp Med. 2016;11(1):21-6.

6. Tärnberg M, Nilsson LE, Dowzicky MJ. Antimicrobial activity against a global collection of skin and skin structure pathogens: results from the Tigecycline Evaluation and Surveillance Trial (T.E.S.T.), 2010-2014. Int J Infect Dis. 2016;49:141-8.

7. Viehman JA, Nguyen MH, Doi Y. Treatment options for carbapenem-resistant and extensively drug-resistant Acinetobacter baumannii infections. Drugs. 2014;74(12):1315-33.

8. Boucher HW, Talbot GH, Bradley JS, et al. Bad bugs, no drugs: no ESKAPE! An update from the Infectious Diseases Society of America. Clin Infect Dis. 2009;48(1):1-12.

9. Antibiotic resistance threats in the United States, 2013. Atlanta: Centers for Disease Control and Prevention; 2013.

10. Antibiotic resistance patient safety atlas: data on antibiotic-resistant healthcare-associated infections. Atlanta: Centers for Disease Control and Prevention; 2015.

11. Castanheira M, Mendes RE, Jones RN. Update on Acinetobacter species: mechanisms of antimicrobial resistance and contemporary in vitro activity of minocycline and other treatment options. Clin Infect Dis. 2014;59(Suppl 6):S367-73.

12. Wang P, Bowler SL, Kantz SF, et al. Comparison of minocycline susceptibility testing methods for carbapenem-resistant Acinetobacter baumannii. J Clin Microbiol. 2016;54(12):2937-41.

13. Petraitis V, Petraitiene R, Maung BW et al. Pharmacokinetics and tissue distribution of minocycline following intravenous administration in rabbits [poster no. 804]. In: IDWeek; 2017.

14. Tarazi Z, Sabet M, Dudley MN, et al. Pharmacodynamics of intravenous minocycline against Acinetobacter baumannii in a rat pneumonia model [poster]. In: Making a Difference in Infectious Diseases 19th Annual Meeting; 2016.

15. Zhou J, Ledesma KR, Tam VH. In vivo efficacy of minocycline for infections caused by Acinetobacter baumannii [abstract]. In: ICAAC; 2015.

16. Bowker KE, Noel AR, Tomaselli SG, et al. Pharmacodynamics of minocycline against Acinetobacter baumannii studied in a pharmacokinetic model of infection [abstract no. A040]. In: ICAAC; 2014.

17. Alfouzan WA, Noel AR, Bowker KE, et al. Pharmacodynamics of minocycline against Acinetobacter baumannii studied in a pharmacokinetic model of infection. Int J Antimicrob Agents. 2017. https://doi.org/10.1016/j.ijantimicag.2017.06.026.

18. Guerra AD, Andes D, Hematti P, et al. Minocycline enhances the antimicrobial activity of unpolarized macrophages against Acinetobacter baumannii while inducing an anti-inflammatory profile [poster]. In: IDWeek; 2017.

19. Hoban DJ, Reinert RR, Bouchillon SK, et al. Global in vitro activity of tigecycline and comparator agents: Tigecycline Evaluation and Surveillance Trial 2004-2013. Ann Clin Microbiol Antimicrob. 2015;14(1):27.

20. Bouchillon SK, Hackel M, Hoban D, et al. Evaluating multi-drug resistant Acinetobacter baumannii in critical care units [abstract no. K9 plus poster]. Am J Respir Crit Care Med. 2011;183:A5830. 
21. Flamm RK, Castanheira M, Streit JM, et al. Minocycline activity tested against Acinetobacter baumannii complex, Stenotrophomonas maltophilia, and Burkholderia cepacia species complex isolates from a global surveillance program (2013). Diagn Microbiol Infect Dis. 2016;85(3):352-5.

22. Denys GA, Callister SM, Dowzicky MJ. Antimicrobial susceptibility among Gram-negative isolates collected in the USA between 2005 and 2011 as part of the Tigecycline Evaluation and Surveillance Trial (T.E.S.T.). Ann Clin Microbiol Antimicrob. 2013;12:24

23. Kehl SC, Dowzicky MJ. Global assessment of antimicrobial susceptibility among Gram-negative organisms collected from pediatric patients between 2004 and 2012: results from the Tigecycline Evaluation and Surveillance Trial. J Clin Microbiol. 2015;53(4):1286-93.

24. Sahm D, Biedenbach D, Leister-Tebbe H. Antimicrobial susceptibility profiles for multi-drug resistant (MDR) Acinetobacter spp: a global perspective (TEST 2011-2014) [abstract no. C-1016 plus poster]. In: ICAAC; 2015.

25. Bowers DR, Cao H, Zhou J, et al. Assessment of minocycline and polymyxin B combination against Acinetobacter baumannii. Antimicrob Agents Chemother. 2015;59(5):2720-5.

26. He $\mathrm{S}, \mathrm{He} \mathrm{H}$, Chen $\mathrm{Y}$, et al. In vitro and in vivo analysis of antimicrobial agents alone and in combination against multi-drug resistant Acinetobacter baumannii. Front Microbiol. 2015;6:507.

27. Rodriguez $\mathrm{CH}$, Nastro $\mathrm{M}$, Vay $\mathrm{C}$, et al. In vitro activity of minocycline alone or in combination in multidrug-resistant Acinetobacter baumannii isolates. J Med Microbiol. 2015;64(10):1196-200.

28. Liang W, Liu XF, Huang J, et al. Activities of colistin- and minocycline-based combinations against extensive drug resistant Acinetobacter baumannii isolates from intensive care unit patients. BMC Infect Dis. 2011;11:109.

29. Yang YS, Lee Y, Tseng KC, et al. In vivo and in vitro efficacy of minocycline-based combination therapy for minocycline-resistant Acinetobacter baumannii. Antimicrob Agents Chemother. 2016;60(7):4047-54.

30. Huang D, Yu B, Diep JK, et al. In vitro assessment of combined polymyxin B and minocycline therapy against Klebsiella pneumoniae carbapenemase (KPC)-producing $K$. pneumoniae. Antimicrob Agents Chemother. 2017:61(7). https://doi.org/10. 1128/AAC.00073-17.

31. Zhou J, Ledesma KR, Chang KT, et al. Pharmacokinetics and pharmacodynamics of minocycline against Acinetobacter baumannii in a neutropenic murine pneumonia model. Antimicrob Agents Chemother. 2017;61(5). https://doi.org/10.1128/AAC. 02371-16.

32. Vilacoba E, Almuzara M, Gulone L, et al. Emergence and spread of plasmid-borne tet(B):ISCR2 in minocycline-resistant Acinetobacter baumannii isolates. Antimicrob Agents Chemother. 2013;57(1):651-4.

33. Vilacoba E, Almuzara M, Gulone L, et al. Widespread dispersion of the resistance element tet(B):ISCR2 in XDR Acinetobacter baumannii isolates. Epidemiol Infect. 2016;144(7):1574-8.

34. Lomovskaya O, Sun D, Rubio-Aparicio D, et al. TetB testing and its absence identifies minocycline (MINO) susceptible isolates of Acinetobacter baumannii (ACB) [poster no. 2043]. In: IDWeek; 2016.

35. Wang P, McElheny CL, Mettus RT, et al. Contribution of TetB efflux pump on minocycline susceptibility among carbapenemresistant Acinetobacter baumannii. Antimicrob Agents Chemother. 2017;61(10). https://doi.org/10.1128/AAC.01176-17.

36. Lomovskaya O, Nelson KJ, Rubio-Aparicio D, et al. Minocycline activity is enhanced by polymyxin $\mathrm{B}$ in tet $B$-containing isolates of Acinetobacter baumannii [poster no. 2041]. In: IDWeek; 2016.
37. Rumbo C, Gato E, López M, et al. Contribution of efflux pumps, porins, and $\beta$-lactamases to multidrug resistance in clinical isolates of Acinetobacter baumannii. Antimicrob Agents Chemother. 2013;57(11):5247-57.

38. Lomovskaya O, Sun D, Rubio-Aparicio D, et al. Accumulation of several chromosomal mutations have limited impact on the sensitivity of Acinetobacter baumannii (ACB) to minocycline (MINO) [abstract no. C-1009 plus poster]. In: ICAAC; 2015.

39. Lomovskaya O, Sun D, King P, et al. Tigecycline (TIG) but not minocycline (MINO) readily selects for clinically relevant effluxmediated resistance (R) in Acinetobacter spp. (ACB) [abstract no. C1-1087 plus poster]. In: ICAAC; 2013.

40. Goff DA, Bauer KA, Mangino JE. Bad bugs need old drugs: a stewardship program's evaluation of minocycline for multidrugresistant Acinetobacter baumannii infections. Clin Infect Dis. 2014;59(Suppl 6):S381-7.

41. Bishburg E, Shah M, Chan T. Use of intravenous minocycline for the treatment of methicillin-resistant Staphylococcus aureus (MRSA) and resistant Gram-negative organisms: experience in a tertiary care hospital. Infect Dis Clin Pract. 2014;22(1):26-31.

42. Wood GC, Hanes SD, Boucher BA, et al. Tetracyclines for treating multidrug-resistant Acinetobacter baumannii ventilatorassociated pneumonia. Intensive Care Med. 2003;29(11):2072-6.

43. Pogue JM, Neelakanta A, Mynatt RP, et al. Carbapenem-resistance in Gram-negative bacilli and intravenous minocycline: an antimicrobial stewardship approach at the Detroit Medical Center. Clin Infect Dis. 2014;59(Suppl 6):S388-93.

44. Chan JD, Graves JA, Dellit TH. Antimicrobial treatment and clinical outcomes of carbapenem-resistant Acinetobacter baumannii ventilator-associated pneumonia. J Intensive Care Med. 2010;25(6):343-8.

45. Khatri A, Lee L, Wang G, et al. Minocycline in treatment of carbapenem-resistant Klebsiella pneumoniae [poster no. 364]. In: IDWeek; 2017.

46. Falagas ME, Vardakas KZ, Kapaskelis A, et al. Tetracyclines for multidrug-resistant Acinetobacter baumannii infections. Int $\mathrm{J}$ Antimicrob Agents. 2015;45(5):455-60.

47. Fan W, Sulham K. Real-world utilization of minocycline: a retrospective database analysis [poster]. In: Making a Difference in Infectious Diseases 19th Annual Meeting; 2016.

48. Charlson ME, Pompei P, Ales KL, et al. A new method of classifying prognostic comorbidity in longitudinal studies: development and validation. J Chronic Dis. 1987;40(5):373-83.

49. Haghi-Aminjan $\mathrm{H}$, Asqhari $\mathrm{MH}$, Goharbari $\mathrm{H}$, et al. A systematic review on potential mechanisms of minocycline in kidney diseases. Pharmacol Rep. 2017;69(4):602-9.

50. Dai C, Ciccotosto GD, Cappai R, et al. Minocycline attenuates colistin-induced neurotoxicity via suppression of apoptosis, mitochondrial dysfunction and oxidative stress. J Antimicrob Chemother. 1. 2017;72(6):1635-45.

51. Lodise TP, Fan W, Griffith DC, et al. Minocycline but not tigecycline is associated with a reduction in colistin-associated acute renal failure in critically ill adult patients [poster]. In: ASM Microbe; 2017.

52. Lodise TP, Fan W, Griffith DC, et al. Coadministration of minocycline with colistin in critically ill patients is associated with reduced incidence of acute renal failure [poster]. In: 27th ECCMID; 2017.

53. Thaden JT, Pogue JM, Kaye KS. Role of newer and re-emerging older agents in the treatment of infections caused by carbapenemresistant Enterobacteriaceae. Virulence. 2017;8(4):403-16.

54. Kaye KS, Pitman RJ, Fan W, et al. Estimating the cost-effectiveness of minocycline for the treatment of multidrug resistant Acinetobacter baumannii [poster no. 2059]. In: IDWeek; 2016.

55. Greig SL, Scott LJ. Intravenous minocycline: a review in Acinetobacter infections. Drugs. 2016;76(15):1467-76. 